\title{
A "word length effect" for sign language: Further evidence for the role of language in structuring working memory
}

\author{
MARGARET WILSON and KAREN EMMOREY \\ The Salk Institute for Biological Studies, La Jolla, California
}

\begin{abstract}
We report a sign length effect in deaf users of American Sign Language that is analogous to the word length effect for speech. Lists containing long signs (signs that traverse relatively long distances) produced poorer memory performance than did lists of short signs (signs that do not change in location). Further, this length effect was eliminated by articulatory suppression (repetitive motion of the hands), and articulatory suppression produced an overall drop in performance. The pattern of results, together with previous findings (Wilson \& Emmorey, 1997), provides evidence for a working memory system for sign language that consists of a phonological storage buffer and an articulatory rehearsal mechanism. This indicates a close equivalence of structure between working memory for sign language and working memory for speech. The implications of this equivalence are discussed.
\end{abstract}

Our previous research (Wilson \& Emmorey, 1997, described below) has uncovered what appears to be a "phonological loop" for sign language. The phonological loop, as studied in hearing subjects, is a component of working memory that is used for the temporary maintenance of information in a speech-based form (see, e.g., Baddeley, 1986). Our findings have indicated a similar mechanism in the working memory of deaf native users of American Sign Language (ASL). This mechanism is used for the temporary maintenance of sign language materials and seems to be largely parallel in structure to the phonological loop for speech. The data suggest the existence of a storage buffer that is accessed directly through sign presentation and indirectly through picture presentation, the latter case apparently mediated by a manual articulatory rehearsal process.

These findings have implications for our understanding of the architecture of working memory. They indicate that the type of speech-based memory mechanism observed in hearing subjects is not an innate property of the working memory system, based, perhaps, on the evolutionary relationship between language and the vocal and auditory systems. Rather, it suggests that the structure of the phonologi-

This work was supported by NIH Grant DC-00128-01, awarded to M.W., NIH Grant HD-13249, awarded to K.E. and U. Bellugi, as well as NIH Grants DC-00146, DC-0021, and HD-26022. We thank Brenda Falgier and Kevin Clark for help in developing stimuli and running subjects, Diane Brentari for help in the syllabic analysis of our stimuli, and Robert Greene and one anonymous reviewer for their comments and suggestions. We thank Dennis Galvan for his help in recruiting subjects. We are especially grateful to Gallaudet University, Washington, D.C., California State University, Northridge, and the deaf subjects who participated in these studies. Correspondence concerning this article should be addressed to M. Wilson, The Salk Institute for Biological Studies, 10010 North Torrey Pines Rd., La Jolla, CA 92037 (e-mail: wilson@ crl.ucsd.edu)

-Accepted by previous editor, Geoffrey R. Loftus cal loop arises in response to developmental experience, in whichever modality receives the appropriate form of input.

However, our findings so far represent only a partial replication of the body of evidence that has been used to construct the theory of the phonological loop. In the present paper, we offer additional evidence for the structure of signbased working memory. This evidence addresses the word length effect, a major line of evidence that supports Baddeley's model of speech-based working memory. The results of the present investigation strengthen the claim that a phonological loop of approximately equivalent structure can arise in either of two very distinct sensorimotor modalities.

\section{The Evidence for the \\ Speech-Based Phonological Loop}

Much of the evidence for the structure of the phonological loop rests on the phonological similarity effect and the word length effect (see Baddeley, 1986, for a review). In the similarity effect, lists of similar-sounding items produce lower memory performance than do lists of distinctive items (see, e.g., Baddeley, 1966; Conrad \& Hull, 1964). This reduced performance presumably occurs because of confusions between items, and this indicates that the surface form of the language is being retained. In the length effect, lists of long words produce lower memory performance than do lists of short words (Baddeley, Thomson, \& Buchanan, 1975). This effect is presumed to occur because of the involvement of articulatory processes, which places a time limit on the amount of material that can be retained.

Further evidence comes from the effect of suppressing the subject's vocal articulatory processes. This is done by having the subject repeat a word or a syllable continuously during presentation of the stimuli, thus preventing the articulatory mechanisms from being used for task-relevant purposes. This manipulation reduces memory span (see, e.g., Murray, 1968). 
Furthermore, the effect of suppression interacts with the effects of phonological similarity and word length. Specifically, with the visual presentation of stimuli (print or namable pictures), suppression renders the similarity and word length manipulations ineffective. This strongly suggests that the similarity and length effects are produced by articulatory mechanisms (Baddeley, Lewis, \& Vallar, 1984). However, there is an important difference between the similarity effect and the length effect: They are subserved by articulation in different ways.

The similarity effect is only disrupted by articulatory suppression when a speech code must be derived from visual information, such as print or pictures. When words are presented directly as speech, the similarity effect remains robust even under articulatory suppression (Baddeley et al., 1984). The similarity effect is, thus, apparently a product of a nonarticulatory phonological buffer, and articulation is involved only as a means of translating the task materials into a phonological code. When this translation cannot take place, the phonological representation will be absent, and there will be no similarity effect. But if the stimuli are presented directly in phonological form, the similarity effect will occur even when articulation is blocked.

In contrast, the length effect is disrupted by articulatory suppression, regardless of how the stimuli are presented. This suggests that the length effect is a direct product of the articulatory processes themselves. When the articulatory processes are unavailable, the length effect cannot occur.

Furthermore, if one takes the word length effect as diagnostic of the involvement of articulatory processes, the fact that the length effect occurs even with speech presentation suggests that articulatory processes play a greater role in the phonological loop than just translating from print to phonology. The occurrence of the length effect for speech stimuli, together with the disruption of overall performance level by articulatory suppression, suggests that articulation is an integral part of the memory maintenance system. Baddeley's model states that articulation is used to repeatedly feed information back into the storage buffer before it fades.

We should note that there have been challenges to the claim that the word length effect reflects an articulatory process within the phonological loop (see, e.g., Avons, Wright, \& Pammer, 1994; Bishop \& Robson, 1989). However, a growing body of neuropsychological evidence joins the existing behavioral evidence to implicate speechplanning mechanisms in working memory rehearsal (Belleville, Peretz, \& Arguin, 1992; Cubelli \& Nichelli, 1992; Fiez et al., 1996; Waters, Rochon, \& Caplan, 1992). Furthermore, for the present purposes, we are primarily interested in the pattern of data that has been used to dissociate the locus of the similarity effect from the locus of the word length effect.

\section{Partial Evidence for a}

\section{Sign-Based Phonological Loop}

Previously, we have found that certain of these characteristic effects and interactions hold true for sign-based working memory, as well as for speech-based working memory (Wilson \& Emmorey, 1997). We replicated the finding of previous investigators (Hanson, 1982; Klima \& Bellugi, 1979; Krakow \& Hanson, 1985; Poizner, Bellugi, \& Tweney, 1981) that lists of signs that share handshape are recalled less well by deaf signers than are lists of signs with diverse handshapes-an ASL-based phonological similarity effect. ${ }^{1}$ We also found that simple repetitive movement of the hands during stimulus presentation reduced memory performance - a manual articulatory suppression effect. Furthermore, we found that, when stimuli were presented directly in the phonological form in which they would be rehearsed (i.e., presented as signs), the phonological similarity and articulatory suppression effects were independent of one another, but, when stimuli were presented as namable pictures that must be translated into phonological form, articulatory suppression eliminated the phonological similarity effect. As in the case of spoken language, this pattern of findings suggests that there is an articulatory rehearsal mechanism that is disrupted by the competing movement of the relevant articulators and that this rehearsal mechanism is used to convert nonphonological stimuli into phonological form. It further suggests that this rehearsal mechanism is separate from the storage component responsible for the phonological similarity effect. Thus, our findings so far indicate the existence of a sign loop, with a structure that appears to parallel that of the speech loop.

To consolidate this claim, however, we must demonstrate an additional set of effects. If articulatory processes are involved, they ought to have certain consequences, such as the limitation that articulation rate places on how much material can be rehearsed without loss of information. That is, we ought to be able to find a sign length effect that is parallel to the word length effect for speech. Further, if this sign length effect is indeed articulatory in nature, it ought to be vulnerable to articulatory suppression. That is, articulatory suppression ought to reduce or eliminate the sign length effect. Furthermore, in contrast to our findings with the phonological similarity effect, this reduction of the sign length effect ought to occur even when stimuli are presented directly in phonological form.

In fact, there is some evidence that suggests we may not find such articulation-based effects. Whereas hearing subjects show a correlation between articulation rate and memory span (Hulme, Thomson, Muir, \& Lawrence, 1984), Mayberry and Waters (1990) failed to find such a correlation in deaf subjects. However, there were methodological problems with how articulation rate was measured in that study, which may explain the lack of correlation. Given the important role ascribed to articulatory processes in the phonological loop, it is critical for our claims that we discover whether a sign length effect exists.

\section{METHOD}

Two sets of signs were chosen on the basis of movement type to be long sign stimuli and short sign stimuli. Long signs were chosen 


\section{Long Signs}

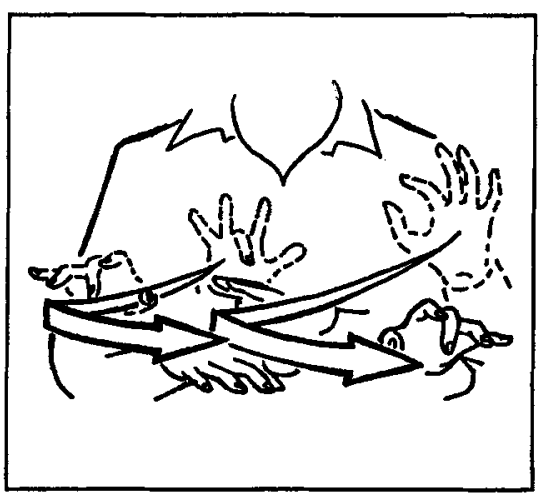

PIANO

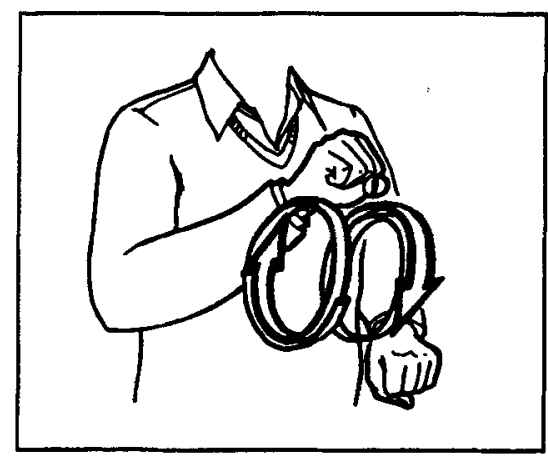

BICYCLE
Short Signs

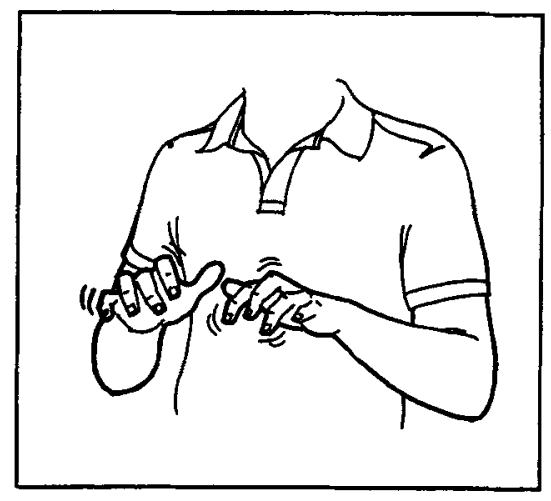

TYPEWRITER

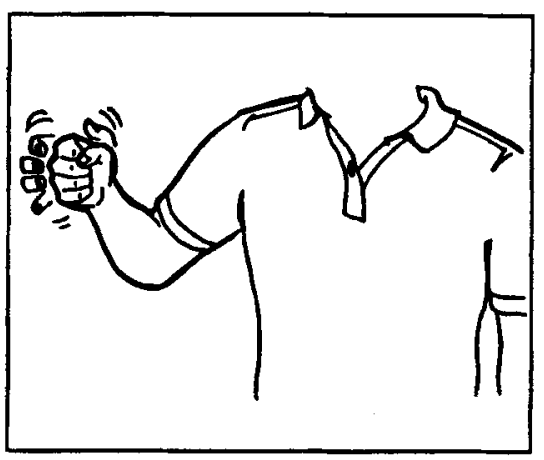

MILK

Figure 1. Illustration of long and short American Sign Language signs, matched for handshape.

to have large circular movement, movement that covered distance, or movement with change of direction. Short signs, in contrast, were chosen to have short movement involving no change of location (e.g., tapping). In addition, pairs of short and long signs were matched for handshape and approximately matched for location (see Figure 1). The two lists were also approximately matched for concreteness of meaning and for length in syllables of their English glosses (see the Appendix). Finally, the two lists were approximately matched in number of monosyllabic and bisyllabic signs (using Brentari's [1990] analysis).

In order to control for the possibility that sign length may have an effect at the time of report, we used a probe paradigm, in which subjects are asked to report only a single item from a particular serial position. (The word length effect has been demonstrated with a probe paradigm by Avons et al., 1994.) Specifically, in the present experiment, the subjects were shown a probe sign after the end of the list and were asked to report the sign that had come immediately after the probe sign. This method allowed us to test for serial memory, while eliminating any effect of report length. Any observed length effect could thus be attributed to the impact of length on retention prior to report. (Experiments on the word length effect in hearing subjects frequently use written reports, in which subjects are allowed to abbreviate long words in order to eliminate report length effectssee, e.g. et al., Baddeley et al., 1984. This solution is not practical here, since the use of written English may bias deaf subjects away from an ASL-based memory strategy-Krakow \& Hanson, 1985.)

Because sign-based memory generally yields lower serial recall performance than does speech-based memory (Hanson, 1982; Klima \& Bellugi, 1979), possibly because of differences in articulation time (Klima \& Bellugi, 1979; Marschark, 1996), the present experiment used relatively few items per list. Pilot experiments indicated that five signs per list with a probe paradigm avoided both ceiling and floor performance for most subjects.

\section{Subjects}

The experiment included 12 deaf students at Califomia State University, Northridge, and at Gallaudet University, Washington, D.C., ranging in age from 19 to 30 years, with a mean age of 24 . All subjects had hearing loss of $80 \mathrm{~dB}$ or greater, and all considered ASL to be their primary language. Of these, 7 had deaf parents or an older deaf sibling and had acquired ASL from birth, whereas the remaining subjects all had learned ASL by age six. Two additional subjects were tested but were excluded because of performance near chance ( $25 \%$ correct).

\section{Stimuli}

Thirty-two lists of 5 signs each were created from a set of 10 long signs, with no sign occurring more than once per list. Likewise, 32 


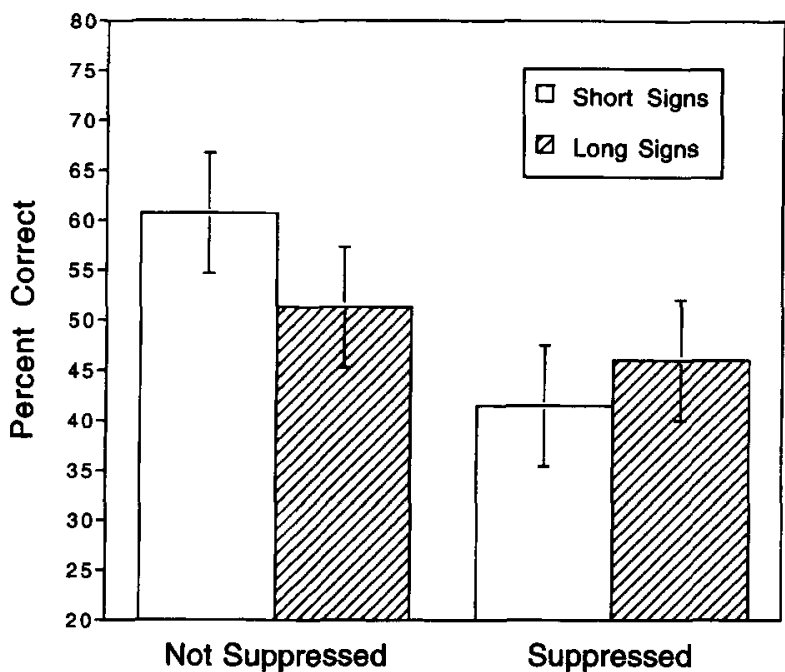

Figure 2. Mean performance on probed recall of American Sign Language signs. Error bars represent within-subjects 95\% confidence intervals.

lists were created from a set of 10 short signs. (English glosses of the long and short signs are listed in the Appendix.) In addition, 8 lists of each length were created for practice, with each stimulus sign used exactly four times. Each sign was filmed separately, beginning and ending with the signer's hands in the lap. These stimuli were cropped to a length of $1.25 \mathrm{sec}$ each and were concatenated into lists with zero interstimulus interval (ISI) by digital editing software (Adobe Premiere) before being transferred to videotape. Stimulus presentation for each trial consisted of a list of signs, followed by a 2-sec retention interval, followed by a single sign from the list (the probe). In all four conditions, probes were chosen with equal frequency from serial positions $1-4$ in the list. (After 4 subjects were run, an error was detected in the balancing of frequency of serial position. For these subjects, eight trials were thrown out to achieve balance, and the stimulus tape was redesigned to correct the balancing for the remaining subjects.)

\section{Design and Procedure}

Two within-subjects factors were tested: suppression (not suppressed vs. suppressed) and length (short vs. long). Trials from all four conditions were mixed, and a break was provided halfway through the experiment. Instructions, which were given in ASL, requested that the subjects produce the sign that had occurred immediately after the probe sign in the stimulus list. The stimuli were presented on videotape by a Panasonic monitor/recorder AG-513. After the presentation of each trial, the experimenter paused the videotape while the subject responded. Suppressed trials were preceded by a blue dot in the upper right corner of the screen for $1 \mathrm{sec}$, which was the subject's cue to begin manual suppression. This consisted of forming the ASL 8 handshape (thumb touches middle finger) with both hands, combined with the movement from the sign wORLD (the two hands circle one another, with contact at the end of each repetition). ${ }^{2}$ This activity was to begin before stimulus presentation and to continue throughout stimulus presentation, retention interval, and probe presentation. If the subjects interrupted the suppression movement or slowed to a rate of less than approximately one repetition per second, they were corrected after the end of the trial. (In fact, all such instances of subjects being corrected occurred during practice.)

\section{RESULTS}

Performance for the four conditions is depicted in Figure 2. Error bars show within-subjects confidence intervals - that is, confidence intervals with intersubject variance removed (Loftus \& Masson, 1994). Inspection of the graph suggests that suppression reduces performance and also that length and suppression interact. Figure 3, which plots difference scores, shows the effect of length for the not-suppressed and suppressed conditions. The confidence interval for the not-suppressed condition lies entirely above zero, suggesting that short sign performance is in fact better than long sign performance. These observations are borne out by statistical tests. A two-way repeated measures analysis of variance did not show a main effect of length $\left[F(1,11)=0.58\right.$, n.s., $\left.M S_{\mathrm{e}}=121.58\right]$ but did show a main effect of suppression $\left[F(1,11)=6.23, M S_{\mathrm{e}}=291.29\right.$, $p<.05]$ and a significant interaction $\left[F(1,11)=6.48, M S_{\mathrm{e}}=\right.$ $90.30, p<.05]$. In the not-suppressed condition, the effect of length was significant $[t(11)=2.08, p<.05$, one tailed]. In the suppressed condition, the apparent reversal of the length effect was not significant $[t(11)=1.17$, n.s.], although this may reflect insufficient power.

\section{DISCUSSION}

These results demonstrate a sign length effect in ASLbased working memory that parallels the word length effect for spoken language. The results also replicate our previous finding that manual articulatory suppression disrupts working memory for signs (Wilson \& Emmorey, 1997). Furthermore, just as with the word length effect for speech, articulatory suppression reduces or eliminates the sign length effect, suggesting that the length effect is articulatory in origin. Given that the probe paradigm used here prevents any effect of length caused by the process of report, the sign length effect appears to be due to the time limitation imposed by an articulatory rehearsal process. This set of findings supports the claim that there is a close equivalence of structure between the phonological loop for speech and the phonological loop for sign.

Inspection of the mean difference scores for the suppressed condition in Figure 3 actually shows slightly better memory for long signs than for short signs. One possible explanation for this reversal, if it is real, is that the hand motion during suppression is similar to that of two of the long signs (and none of the short signs). Compatibility between the suppressing movement and the movement of these signs may have allowed a certain amount of rehearsal to take place, even under suppression. This would result in a slight advantage for the long signs relative to the short signs, which would be more effectively suppressed. This explanation is consistent with the phonological loop model we are invoking to explain working memory for signs.

Finally, we should also note that the interaction between length and suppression, which is predicted by the claim 


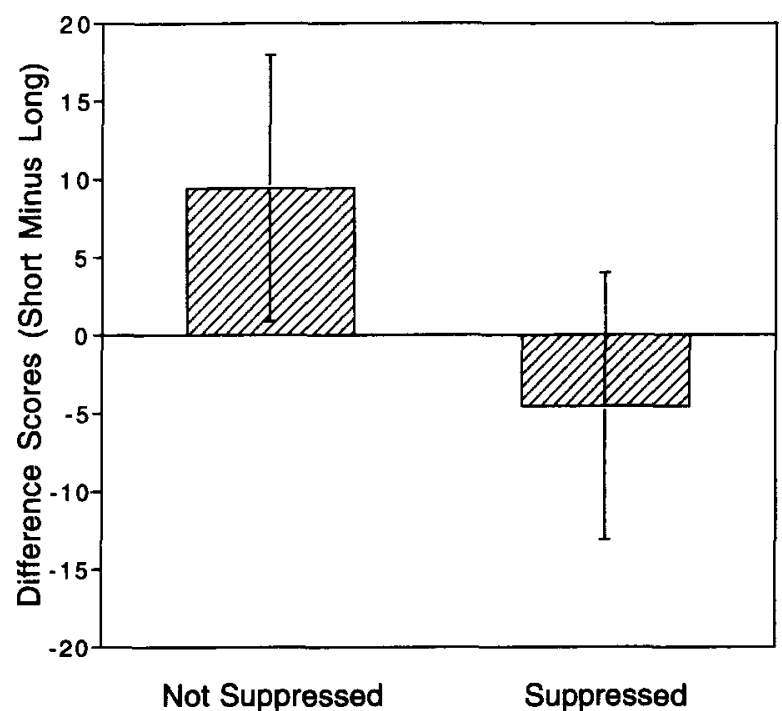

Figure 3. Difference scores (short sign condition minus long sign condition) for the not-suppressed and suppressed conditions. Error bars represent within-subjects $95 \%$ confidence intervals.

that the length effect is articulatory, would not be expected if the observed "length effect" were in fact a spurious result of some other unintended difference between the two sets of signs. Thus, the observed pattern of results validates the choice of short and long sign stimuli.

\section{The Nature of Articulatory Rehearsal}

A controversy exists in the literature over whether the word length effect results only from differences in length that are specified in the phonological representation (e.g., difference in number of syllables) or whether it can arise from factors that affect length because of the constraints of articulation itself (e.g., English long vs. short vowels or articulatorily simple vs. complex combinations of phonemes). The present results may bear on this issue. Although Baddeley, Thomson, and Buchanan (1975) found a word length effect for long as opposed to short words that were matched for number of syllables and phonemes and that differed only in the articulation length of the phonemes themselves, Caplan, Rochon, and Waters (1992) failed to replicate this result. Caplan et al. (p. 186) argue that, although word length effects (e.g., those based on number of syllables) do "require some form of phonological output planning," this output planning does not fully specify articulatory gestures. Instead, they conceive of this output planning as activating "underspecified lexical phonological representations (p. 189)." (See Baddeley \& Andrade, 1994; Caplan \& Waters, 1994, for further debate on this issue.)

Our findings on the sign length effect may pose a challenge to the view proposed by Caplan et al. (1992). Unlike spoken language, additional syllables in sign language do not necessarily add additional articulatory length, according to most accounts of ASL syllable structure (for reviews, see Brentari, 1995; Corina \& Sandler, 1993). Indeed, our set of long signs contained a few monosyllabic signs (e.g., LANGUAGE, YEAR), whose matched short signs were bisyllabic (e.g., LICENSE, STATISTICS). Thus, the features that differentiate our long and short signs apparently affected articulation time for reasons related to the articulatory gestures themselves (e.g., distance to be traveled) rather than for reasons related to the phonological representation. For speech, the types of length manipulations used by Baddeley et al. (1975) and by Caplan et al. (e.g., long vs. short vowels) may simply not result in large enough length differences to create a robust effect. In contrast, because sign language involves large scale movement, it may be much easier to construct sizable length differences without resorting to adding syllables, thus providing a better test of whether length effects can be found for relatively peripheral features of the stimuli. Our data seem to argue that peripheral features of language output are indeed involved in the phonological loop.

\section{The Interplay of Language \\ Structure and Sensory Processing}

The results of the present study indicate that articulatory processes play a similar role in sign-based and speechbased working memory, lending support to our previous claim for a sign-based "phonological loop" that parallels the speech-based loop in structure (Wilson \& Emmorey, 1997). We should note, however, that not all of our findings indicate complete equivalence between the speech loop and the sign loop. In one study we found that deaf children who were native signers of ASL were approximately equal on forward and backward digit span, in striking contrast to the standard finding of severely diminished performance on backward span relative to forward span for hearing children (Wilson, Bettger, Niculae, \& Klima, 1997). This result can be interpreted in terms of information-processing differences between the auditory and visual modalities. Whereas audition tends to code order information temporally, and hence unidirectionally, vision can code order information spatially, without a necessary directionality. Thus, differences between audition and vision may create differences between the speech and sign loops in terms of exactly how information is represented in the phonological storage buffer.

Nevertheless, our previous (Wilson \& Emmorey, 1997) and present results indicate that the basic configuration of components and how they interact is the same for the speech and sign loops. This claim has implications for our understanding of how working memory develops. The structure of the phonological loop appears not to be predetermined by the evolutionary relationship between language and the auditory/vocal modality. Instead, it appears to develop in response to language experience, regardless of sensorimotor modality. 
This raises the possibility that similar rehearsal loop structures could arise for other forms of expertise besides language, such as musical expertise. The apparently critical role of articulatory processes in both the speech loop and the sign loop suggests that a necessary precondition for such rehearsal loops may be a tight, overlearned relationship between input and output representations. It may be that imitatability or rehearsability is a hallmark of the type of information that will allow for this type of memory mechanism. In that case, we would indeed expect such a mechanism for expert musicians, who have overlearned the relationship between certain notes (or relationships and combinations of notes) and certain motoric patterns involved in playing an instrument.

A similar suggestion regarding the role of learned motor patterns in the creation of rehearsal mechanisms has been made by Reisberg, Rappaport, and O'Shaughnessy (1984; see also Reisberg \& Logie, 1993). Although the motoric system for representing information that was invented by Reisberg et al. was extremely simple (capable of representing only the numbers 1-10 and practiced by subjects for only a few hours), it nevertheless hints at both the importance of articulation as a form of representation in working memory and the flexibility of working memory in its ability to acquire and exploit novel representational systems. These conclusions fit well with the implications of the present findings. The articulatory and phonological properties of a language apparently are sufficient to give rise to a rehearsal loop structure within working memory, regardless of the sensorimotor modality of the language.

\section{REFERENCES}

Avons, S. E., Wright, K. L., \& Pammer, K. (1994). The word-length effect in probed and serial recall. Quarterly Journal of Experimental Psychology, 47A, 207-231.

BADDELEY, A. D. (1966). Short-term memory for word sequences as a function of acoustic, semantic and formal similarity. Quarterly Journal of Experimental Psychology, 18, 362-365.

Baddeley, A. (1986). Working memory. Oxford: Oxford University Press. BADDELEY, A., \& ANDRADE, J. (1994). Reversing the word-length effect: A comment on Caplan, Rochon, \& Waters. Quarterly Journal of Experimental Psychology, 47A, 1047-1054

Baddeley, A., Lewis, V. J., \& Vallar, G. (1984). Exploring the articulatory loop. Quarterly Journal of Experimental Psychology, 36A, 233-252.

Baddeley, A., Thomson, N., \& Buchanan, M. (1975). Word length and the structure of short-term memory. Journal of Verbal Learning \& Verbal Behavior, 14, 575-589.

Belleville, S., Peretz, I., \& Arguin, M. (1992). Contribution of articulatory rehearsal to short-term memory: Evidence from a case of selective disruption. Brain \& Language, 43, 713-746.

Bellugi, U., Klima, E. S., \& SiPle, P. (1975). Remembering in signs. Cognition, 3, 93-125.

BisHop, D. V. M., \& RoBson, J. (1989). Unimpaired short-term memory and rhyme judgement in congenitally speechless individuals: Implications for the notion of "articulatory coding." Quarterly Journal of Experimental Psychology, 41A, 123-140.

Brentari, D. (1990). Theoretical foundations of American Sign Language phonology. Unpublished doctoral dissertation. University of Chicago, Chicago.
BRENTARI, D. (1995). Sign language phonology: ASL. In J. Goldsmith (Ed.), Handbook of phonological theory (pp. 615-639). Oxford: Blackwell.

Caplan, D., Rochon, E., \& Waters, G. S. (1992). Articulatory and phonological determinants of word length effects in span tasks. Quarterly Journal of Experimental Psychology, 45A, 177-192.

CAPLAN, D., \& WATERS, G. S. (1994). Articulatory length and phonological similarity in span tasks: A reply to Baddeley and Andrade. Quarterly Journal of Experimental Psychology, 47A, 1055-1062.

ConRaD, R., \& Hull, A. J. (1964). Information, acoustic confusion and memory span. British Journal of Psychology, 55, 429-432.

CORINA, D., \& SANDLER, W. (1993). On the nature of phonological structure in sign language. Phonology, 10, 165-207.

Coulter, G. R. (Ed.) (1993). Phonetics and phonology: Current issues in ASL phonology. San Diego, CA: Academic Press.

Cubelli, R., \& Nichelli, P. (1992). Inner speech in anarthria: Neuropsychological evidence of differential effects of cerebral lesions on subvocal articulation. Journal of Clinical \& Experimental Neuropsychology, 14, 499-517.

Fiez, J. A., Raife, E. A., Balota, D. A., Schwarz, J. P., Raichle, M. E., \& PETERSEN, S. E. (1996). A positron emission tomography study of the short-term maintenance of verbal information. Journal of Neuroscience, 16, 808-822.

HANSON, V. L. (1982). Recall of order information by deaf signers: Phonetic coding in temporal order recall. Memory \& Cognition, 18, 604-610.

Hulme, C., Thomson, N., Muir, C., \& Lawrence, A. (1984). Speech rate and the development of short-term memory span. Journal of Experimental Child Psychology, 38, 241-253.

KLIMA, E. S., \& Bellugi, U. (1979). The signs of language. Cambridge, MA: Harvard University Press.

KRAKOW, R. A., \& Hanson, V. L. (1985). Deaf signers and serial recall in the visual modality: Memory for signs, fingerspelling, and print. Memory \& Cognition, 13, 265-272.

LoFTus, G. R., \& Masson, M. E. J. (1994). Using confidence intervals in within-subject designs. Psychonomic Bulletin \& Review, 1, 476-490.

MARSCHARK, M. (1996, November). Influences of signed and spoken language on memory span. Paper presented at the 37th Annual Meeting of the Psychonomic Society, Chicago.

MAYBERRY, R. I., \& WATERS, G. S. (1990). Children's memory for sign and fingerspelling in relation to production rate and sign language input. In P. Siple \& S. Fischer (Eds.), Theoretical issues in sign language research: Psychology (pp. 211-229). Chicago: University of Chicago Press.

MURRAY, D. J. (1968). Articulation and acoustic confusability in shortterm memory. Journal of Experimental Psychology, 78, 679-684.

Poizner, H., Bellugi, U., \& Tweney, R. (1981). Processing of formational, semantic, and iconic information in American Sign Language. Journal of Experimental Psychology: Human Perception \& Performance, 7, 1146-1159.

REISBERG, D., \& LOGIE, R. (1993). The ins and outs of working memory: Overcoming the limits on learning from imagery. In B. RoskosEwoldson, M. J. Intons-Peterson, \& R. E. Anderson (Eds.), Imagery, creativity, and discovery: A cognitive perspective (pp. 39-76). New York: Elsevier.

REISBERG, D., RAPPAPORT, I., \& O'ShAughnesSy, M. (1984). Limits of working memory: The digit digit-span. Journal of Experimental Psychology: Learning, Memory, \& Cognition, 10, 203-221.

SANDLER, W. (1995). One phonology or two? Sign language and phonological theory. Glot International, 1, 3-8.

WATERS, G. S., Rochon, E., \& CAPLAN, D. (1992). The role of highlevel speech planning in rehearsal: Evidence from patients with apraxia of speech. Journal of Memory \& Language, 31, 54-73.

Wilson, M., Bettger, J. G., Niculae, I., \& Klima, E. S. (1997). The impact of language modality on the structure of working memory. Journal of Deaf Studies \& Deaf Education, 2, 152-162.

WILSON, M., \& EMMOREY, K. (1997). A visuospatial "phonological loop" in working memory: Evidence from American Sign Language. Memory \& Cognition, 25, 313-320. 


\section{NOTES}

1. The sublexical structure of ASL exhibits abstract phonological properties, such as hierarchically organized feature classes, autosegmental representations, deletion and segmentation rules, a sonority hierarchy, and syllabic structure. See the following references for reviews of ASL phonological structure and for arguments supporting the use of the term phonology to characterize the sublexical structure of signed languages: Brentari (1995), Corina and Sandler (1993), Coulter (1993), and Sandler (1995)

2. Pilot data indicate that this movement is a more effective form of suppression than that used in our previous study (Wilson \& Emmorey, 1997), perhaps because it involves a specified location and a more structured handshape, allowing less possibility for concurrent rehearsal.
APPENDIX

English Glosses of Long and Short Signs

\begin{tabular}{ll}
\hline LONg Signs & Short Signs \\
\hline CROSS & CHURCH \\
BIKE & MILK \\
TOAST & POTATO \\
AMERICA & FOOTBALL \\
LANGUAGE & LICENSE \\
COMMITTEE & COP \\
NAVY & RUSSIA \\
PIE & SCHOOL \\
PIANO & TYPEWRITER \\
YEAR & STATISTICS \\
\hline
\end{tabular}

(Manuscript received October 29, 1996;

revision accepted for publication March 10, 1997.) 